\title{
Hearing screening in schoolchildren: accuracy of different criteria used to analyze transient evoked otoacoustic emissions
} Aryelly Dayane da Silva Nunes-Araújo
https://orcid.org/0000-0002-3814-2675

Sheila Andreoli Balen ${ }^{1}$ https://orcid.org/0000-0003-1353-4362

Antonio Pereira Junior ${ }^{2}$ https://orcid.org/0000-0002-0808-1058 Isabelle Ribeiro Barbosa ${ }^{3}$ https://orcid.org/0000-0002-1385-2849

Universidade Federal do Rio Grande do Norte - UFRN, Laboratório de Inovação Tecnológica em Saúde (LAIS), Hospital Universitário Onofre Lopes, Departamento de Fonoaudiologia, Natal, Rio Grande do Norte, Brasil.

Universidade Federal do Pará - UFPA, Laboratório de Processamento de Sinal, Belém, Pará, Brasil.

Universidade Federal do Rio Grande do Norte - UFRN, Faculdade de Ciências da Saúde do Trairi, Programa de Pósgraduacão em Saúde Coletiva, Santa Cruz, Rio Grande do Norte, Brasil.

Research support source: Coordination for the Improvement of Higher Education Personnel - Brazil (CAPES, as per its Portuguese acronym) - Code 001

Conflict of interests: Nonexistent

\section{(c) (i)}

Received on: March 9, 2021

Accepted on: October 27, 2021

Corresponding address:

Sheila Andreoli Balen

Universidade Federal do Rio Grande do

Norte, Centro de Ciências da Saúde

Rua Gen. Gustavo de Farias, s/n -

Coordenação do Curso de Fonoaudiologia

Petrópolis

CEP: 59020-020 - Natal,

Rio Grande do Norte, Brasi

E-mail: sheila@sheilabalen.com

\section{ABSTRACT}

Purpose: to compare the accuracy of different criteria used to analyze transient evoked otoacoustic emissions in schoolchildren.

Methods: an accuracy study, where an audiological assessment (audiometry, logoaudiometry, tympanometry) and transient emissions were performed with 70 schoolchildren, from the first to the fifth grade of a municipal school, in Northeastern Brazil (6-14 years, $9.9 \pm 2$ years), with four criteria, all with signal-to-noise ratio $\geq 3 \mathrm{~dB}$, being: criterion $A$, in all frequency bands; $B$, in three consecutive frequency bands; $C$, in three of the five non-consecutive frequency bands; D, in 2, 3 and $4 \mathrm{kHz}$. Sensitivity, specificity, accuracy and predictive values with their respective confidence intervals of 95\% were analyzed.

Results: criterion A showed higher sensitivity (92.31\%, 95\% Cl: 67-98\%) and lower specificity (17.35\%, 95\% Cl: 10-29\%); criterion C higher specificity $(84.21 \%, 95 \%$ $\mathrm{Cl}$ : $72-91 \%)$ and higher positive predictive value (52.63\%; 95\% Cl: 51.63-54.63). Accuracy was $82.85 \%$ (95\% Cl 78.23-87.47) in criterion C and 70\% (95\% Cl: $65.38-$ 74.62) in criterion B.

Conclusion: criterion $\mathrm{C}$, signal-to-noise ratio $\geq 3 \mathrm{~dB}$ in three non-consecutive frequency bands, showed the best accuracy, being considered the best choice as a criterion for the isolated use of transient emissions as a hearing screening procedure, in schoolchildren.

Keywords: Data Accuracy; School; Child; Mass Screening; Hearing Tests 


\section{INTRODUCTION}

The worldwide prevalence of disabling hearing loss in children is 34 million cases ${ }^{1}$, being substantially higher in low/middle-income countries compared to high-income ones ${ }^{2}$. In the school age group, the prevalence of hearing loss varies according to sociocultural conditions and access to health services. Childhood hearing impairment may occur after middle ear complications $^{3}$ and may be associated with upper airway infection $^{4}$ and cerumen accumulation as a cause of hearing loss ${ }^{5,6}$. Lack of access to primary health care in developing countries often leads to inadequate treatment of middle ear infections in children.

In view of the hearing risk from middle ear infections and the benefits of early intervention, regular hearing assessment of schoolchildren is recommended ${ }^{7,8}$, preferably within a hearing health program ${ }^{8}$. Although surveys of the prevalence of hearing loss in this population have different methods, the relevance of investigating hearing loss and the need for early intervention in this age group are unanimous ${ }^{9}$. The procedure defined as the reference standard used to assess schoolchildren is pure-tone audiometry ${ }^{7}$, while acoustic immittance measurements and otoscopy are the tests chosen to detect specific middle ear alterations. Transient Evoked Otoacoustic Emissions (TEOAE) can be used in school hearing screening ${ }^{7,10,11}$ because it is a fast procedure with a high rate of acceptance by children, and therefore suitable for school hearing screening programs ${ }^{12}$. In addition, there is evidence that it has better sensitivity and specificity when compared to pure-tone audiometry and tympanometry, with the advantage of being a method that does not require the response of the child and is faster than the others, especially when compared to pure-tone audiometry ${ }^{9}$. Implementing the use of TEOAE may reduce referral rates, increase identification of hearing loss and improve follow-up rates for hearing disorders ${ }^{13}$.

Generally, the use of TEOAE as a hearing screening method in school uses predominantly click stimuli that have a wide frequency band and produce a global cochlear response. By and large, the click stimulus is the standard of TEOAE equipment for hearing screening ${ }^{14}$. The click TEOAE amplitude tends to decrease with age. The amplitude of otoacoustic responses is greatest up to 1 year of age (about $4.25 \mathrm{~dB} \mathrm{SPL}$ ), decreasing to $0.26-0.52 \mathrm{~dB}$ SPL from 1 to 10 years, $0.23 \mathrm{~dB}$ SPL from 11 to 25 years, and $0.14 \mathrm{~dB}$ SPL from 26 to 60 years $^{15}$. The signal-to-noise (SNR) ratio also decreased with increasing age in the frequency bands above 1.5
$\mathrm{kHz}$. The signal-to-noise ratio at higher frequencies reduces faster than at lower frequencies, leading to the maximum change in the signal-to-noise ratio from 2 , 3 and $4.0 \mathrm{kHz}$ in neonates to $1.5 \mathrm{kHz}$ in adults, which further decreases the total TEOAE response level ${ }^{15}$. During development, it is noted that, in neonates, TEOAE responses are most robust in the frequency bands from 2 and $4 \mathrm{kHz}$; and in preschoolchildren and schoolchildren, they are generally uniform between 1 and $4 \mathrm{kHz}$.

In the recommendations of the American Academy of Audiology ${ }^{7}$, the pass/fail criteria for each TEOAE equipment should be established according to the age group served by the hearing screening program. Nevertheless, comparative scientific evidence of the accuracy of different criteria is needed to help professionals who work with hearing screening in schoolchildren using the TEOAE click stimuli ${ }^{16}$, contributing to the use of the procedure as a tool for hearing screening in schoolchildren and, consequently, to the identification of hearing loss.

Based on the changes that occur in the TEOAE responses and the signal-to-noise ratios during development, it is inferred that the TEOAE pass/fail criterion adopted for neonates should not necessarily be the same used in hearing screening in preschoolchildren and schoolchildren. Accordingly, this study aimed at comparing the accuracy of different TEOAE analysis criteria in hearing screening of schoolchildren.

\section{METHODS}

The current study of diagnostic accuracy in the comparison of different TEOAE analysis criteria as a protocol for hearing screening in schoolchildren with audiological assessment was carried out after approval by the Research Ethics Committee of the Federal University of Rio Grande do Norte, Brazil ( $n$ - 030/11) and signing of the Free and Informed Consent Form by parents or guardians of the children.

Based on a population of 182 schoolchildren enrolled in the first to fifth grades in a Municipal School in Natal (RN) and based on an estimated proportion of hearing loss of $16.8 \%^{3}$, a margin of error of $7 \%$ and a non-response rate of $5 \%$, the sample calculation resulted in 72 schoolchildren. Of the 80 students who came to school after being invited, 3 special educational needs and could not finish all the procedures and 7 dropped out. Accordingly, the sample consisted of 70 schoolchildren aged between 6 and 14 years $(9.9 \pm 2)$, 36 males and 34 females. It was founf that $8(11.4 \%)$ 
were enrolled in the first grade, $9(12.8 \%)$ in the second grade, $13(18.6 \%)$ in the third grade, $20(28.6 \%)$ in the fourth grade and $20(28.6 \%)$ in the fifth grade. The eligibility criteria for the sample were: being enrolled and attending school regularly, being able to perform all the procedures and perform them all on the same day, and not revealing an obstruction of the External Acoustic Meatus (EAM) at the time of the assessment.

The schoolchildren were submitted to meatoscopy, pure-tone audiometry (PTA), logoaudiometry, tympanometry and TEOAE, carried out in a quiet room in the school by trained researchers, who assessed each procedure independently, that is, without knowledge of the results of the other procedures, thus characterizing a single-blind study. PTA, logoaudiometry and otoacoustic emissions were performed in a acoustic enclosure. All procedures were performed on the same day to ensure the same assessment conditions in calibrated equipment. The ear at the beginning of the assessment was alternated in all procedures to avoid the effect of the initial ear. If an alteration was detected in any procedure, the schoolchild was immediately retested after removing the earphones or earpieces and re-instructing the procedure ${ }^{7}$. The noise intensity level was measured with an ICEL Manaus DL-4020 decibel meter before, during and at the end of the procedures, with a mean of less than $50 \mathrm{~dB}$. The TEOAE survey was interrupted when the equipment detected excessive environmental noise, and then resumed under favorable acoustic conditions.

Meatoscopy with the Heidji otoscope assessed the conditions of the External Acoustic Meatus (EAM) of both ears; in the presence of obstruction or presence of a foreign body, the schoolchild was excluded from the study. PTA was carried out with an Interacoustic AD229 audiometer and TDH-39 earphones. Hearing thresholds were assessed by airway in the frequencies from 250 to $8000 \mathrm{~Hz}$, and the bone pathway assessment was performed in the frequencies from 500 to $4000 \mathrm{~Hz}$ in the presence of lowered airway thresholds, both by the descending method. Speech recognition threshold (SRT) and speech recognition index (SRI) were investigated in logoaudiometry. Masking was applied when it was possible for the contralateral ear to contribute to the response of the tested ear. The schoolchildren were considered to have normal hearing thresholds when the quadritonal mean $(500,1000,2000$ and $4000 \mathrm{~Hz})$ was equal to or lower than $15 \mathrm{~dB} \mathrm{HL}^{17}$, and the best ear was considered to have SRT equal to or higher than
$92 \%$, SRI compatible with the mean of 500,1000 and $2000 \mathrm{~Hz}^{18}$.

Tympanometry was performed with an Interacoustic AT235 tympanometer in automatic mode, with a probe tone of $226 \mathrm{~Hz}$, generating pressure from +200 to -400 daPa. In order to define the type A tympanometric curve, it was considered a middle ear pressure of 0 daPa or close to it (ranging from +100 to $-100 \mathrm{daPa}$ ) and static compliance between 0.3 and $1.6 \mathrm{cc}^{19}$. The presence of negative pressure above $-150 \mathrm{daPa}$ (type $C$ curve) or absence of pressure variation (type $B$ curve) indicated conductive problems.

In order to capture the transient evoked otoacoustic emissions (TEOAE), the Otoport equipment from Otodynamics in the Quickscreen mode was used, with click stimuli of intensity of $84 \mathrm{dBpe}$ and bandwidth size from 841 to $4757 \mathrm{~Hz}$. The response, in the form of acoustic pressure in the EAM, was captured by a microphone attached to the probe that allowed the separation of Otoacoustic Emissions (OAE) and noise ${ }^{20}$. The amplitudes and the signal-to-noise ratio were recorded in the frequency bands of 1000, 1500, 2000, 3000 and $4000 \mathrm{~Hz}$ for each ear. The TEOAE reproducibility indexes, the level of noise present in the external acoustic meatus during testing, the time required (seconds) to capture TEOAE and the probe stability indexes in EAM were also recorded. In case of absence of TEOAE response, the probes were removed and then retesting was performed immediately, keeping and considering only the last response in the analysis ${ }^{7}$. When audiological alterations were detected in any of the audiological assessment stages, the schoolchild was referred for medical assessment at the Municipal Health Network.

In order to analyze the TEOAE pass criteria, it was established four criteria (Figure 1). In all criteria, it was adopted a $3 \mathrm{~dB}$ signal-to-noise ratio following the suggestion of the equipment used, as well as suggestions from other studies conducted with the same age group ${ }^{14}$. Therefore, the variability studied among the criteria was the number of frequency bands with $3 \mathrm{~dB}$ signal-to-noise ratio (criterion A compared to the other criteria), if they were consecutive or not (criterion B $X$ criterion $C$ ) and frequency specificity (criterion $D$ ). These analysis choices were based on criteria adopted in another study and that may impact the decision making when defining if the individual passed or failed the TEOAE procedure during school hearing screening. All of the pass/fail criteria also met the conditions of TEOAE reproducibility higher than $50 \%$ and probe 
stability higher than $70 \%{ }^{21}$. As a "pass" result, it was considered the presence of TEOAE in both ears in each studied protocol. Accordingly, the data analysis was performed by the number of individuals in the study, and not the number of assessed ears, keeping the logic of pass/fail of the individual in a Hearing Screening Program?.

Figure 1

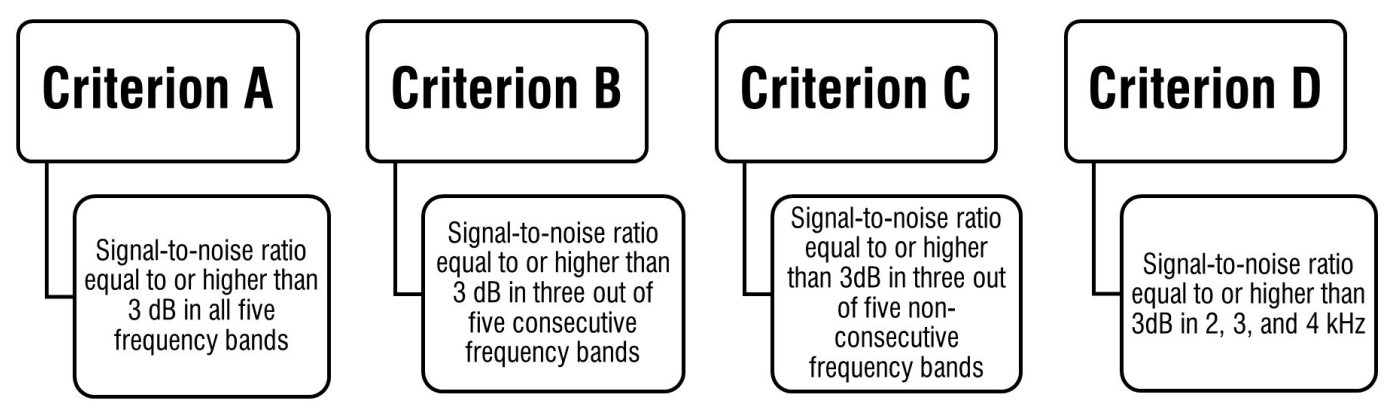

Figure 1. Pass criteria adopted in transient evoked otoacoustic emissions based on the signal-to-noise ratio in the frequency bands

The determination of accuracy is performed by comparing the "sick" and "not sick", thus being classified from the reference test. Sensitivity is defined as the proportion of "sick" individuals that test positive for the disease under study, and specificity as the proportion of "not sick" individuals that test negative for the disease ${ }^{22}$. In this study, individuals identified with hearing loss were considered "sick", while those who did not show hearing loss in the audiological assessment were considered "not sick". In case of a positive result, the positive predictive value is the probability of having the disease; and, in case of a negative result, the negative predictive value is the probability of not having the disease ${ }^{22}$, in this case, hearing loss.

The analysis of the results of pure-tone audiometry, logoaudiometry and tympanometry, which made up the audiological assessment (reference test), allowed the characterization of the presence of hearing loss as to type and degree. The presence or absence of hearing loss was considered as the result of the diagnostic test that was compared to the TEOAE result (index test), and individuals with hearing loss were not excluded, so that it was possible to determine the accuracy of the protocols. It was established the occurrence of a pass/ fail in the TEOAE of the sample for each of the four exposed criteria. From these results, the diagnostic characteristics of each criterion were assessed, comparing its pass/fail result with the presence/absence of hearing loss through the $2 \times 2$ table. Accordingly, the measurements of sensitivity, specificity, accuracy and positive/negative predictive values were obtained for each criterion with their respective confidence intervals of $95 \%$.

\section{RESULTS}

Among the assessed schoolchildren, 57 (81.43\%) had a normal audiological assessment, indicating the absence of hearing loss, and 13 (18.57\%) had hearing loss (HL). With respect to the type of HL, 11 (15.71\%) schoolchildren had conductive hearing loss and 2 (2.85\%) sensorineural. As for laterality, 5 (38.46\%) were bilateral and 8 (61.53\%) unilateral.

The mean TEOAE capture time was 18.98 seconds, with a mean probe stability of $96.85 \%$ and reproducibility of $77.62 \%$. The mean signal-to-noise ratio obtained per ear in the frequency band of $1 \mathrm{kHz}$ was lower than the other frequencies in the TEOAE test (Figure 2).

When analyzing the occurrence of pass/fail according to the studied criteria, criterion $\mathrm{A}$ had the highest occurrence of failure, while criterion $C$ had the highest occurrence of pass. From the analysis of sensitivity and specificity, it was found that criteria $A$ and $D$ had higher sensitivity, while criteria $B$ and $C$ had higher specificity. The PPV of criterion $C$ is higher, while the highest NPV is for criterion A (Table 1). 


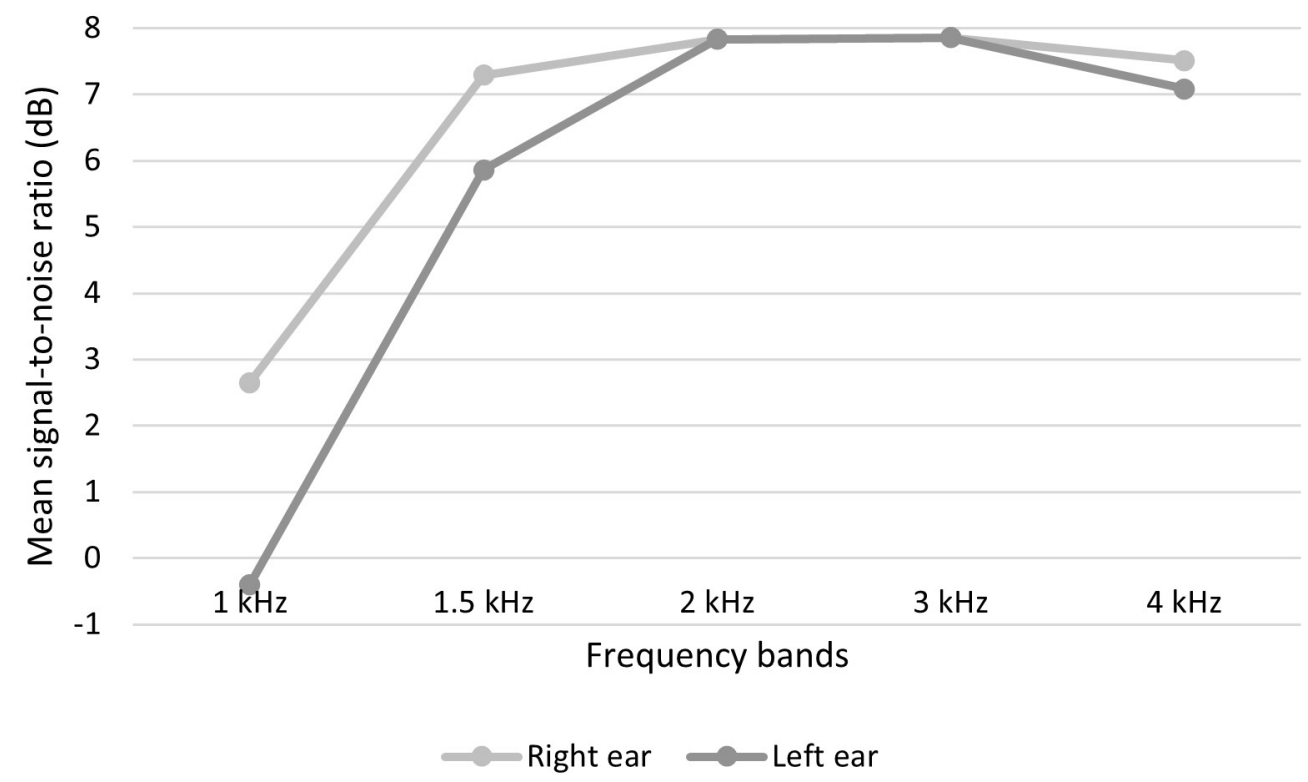

Figure 2. Mean signal-to-noise ratio per ear and frequency bands in the studied sample

Table 1. Occurrence of pass/fail in transient evoked otoacoustic emissions and results of sensitivity, specificity, predictive values and accuracy, according to the analyzed criteria

\begin{tabular}{cccccccc}
\hline Criterion & Pass & Fail & SE & SP & PPV & NPV & Accuracy \\
\hline \multirow{2}{*}{ A } & 11 & 59 & $92.31 \%$ & $17.54 \%$ & $20.34 \%$ & $9.09 \%$ & $31.42 \%$ \\
& $(15.72 \%)$ & $(84.28 \%)$ & $(\mathrm{Cl}: 67-98 \%)$ & $(\mathrm{Cl}: 10-29 \%)$ & $(\mathrm{Cl}: 19.34-31.34 \%)$ & $(\mathrm{Cl}: 8.09-10.09 \%)$ & (Cl: $26.80-36.04 \%)$ \\
B & 42 & 28 & $76.92 \%$ & $68.42 \%$ & $35.71 \%$ & $7.14 \%$ & $70 \%$ \\
& $(60 \%)$ & $(40 \%)$ & $(\mathrm{Cl}: 49-92 \%)$ & $(\mathrm{Cl}: 55-79 \%)$ & $(\mathrm{Cl}: 41.86-44.86 \%)$ & $(\mathrm{Cl}: 13.29-15.29 \%)$ & $(\mathrm{Cl}: 65.38-74.62 \%)$ \\
C & 51 & 19 & $76.92 \%$ & $84.21 \%$ & $52.63 \%$ & $5.88 \%$ & $82.85 \%$ \\
& $(72.86 \%)$ & $(27.14 \%)$ & $(\mathrm{Cl}: 49-92 \%)$ & $(\mathrm{Cl}: 72-91 \%)$ & $(\mathrm{Cl}: 51.63-54.63 \%)$ & $(\mathrm{Cl}: 4.68-6.58 \%)$ & $(\mathrm{Cl}: 78.23-87.47 \%)$ \\
D & 45 & 25 & $84.62 \%$ & $40.35 \%$ & $24.44 \%$ & $8.00 \%$ & $48.57 \%$ \\
& $(64.28 \%)$ & $(35.71 \%)$ & $(\mathrm{Cl}: 57-95 \%)$ & $(\mathrm{Cl}: 28-53 \%)$ & $(23.44-26.44 \%)$ & $(\mathrm{Cl}: 7-9 \%)$ & $(\mathrm{Cl}: 43.95-53.19 \%)$ \\
\hline
\end{tabular}

Captions: SE - sensitivity; SP - specificity; PPV - positive predictive value; NPV - negative predictive value; $\mathrm{Cl}$ - confidence interval.

\section{DISCUSSION}

The results of the current study showed that the choice of assessment criteria influenced the TEOAE accuracy during hearing screening. The criteria $A$ and $D$ proposed by this study resulted in higher sensitivity, while criteria $B$ and $C$ produced higher specificity, the latter being associated with the highest positive and negative predictive values. The accuracy was highest for criterion $\mathrm{C}$, followed by criterion $\mathrm{B}$, while criteria $\mathrm{D}$ and $A$ had an accuracy below $50 \%$.

The SNR ratio is an important parameter in the TEOAE analysis, commonly with more robust responses in neonates ${ }^{16}$. In the school context, TEOAE can be applied in association with other procedures, such as hearing threshold tests ${ }^{6}$, and TEOAE by distortion product ${ }^{23,24}$ or chirp stimuli ${ }^{14}$ can also be used. Due to the fact that the goal of this study was to learn about different TEOAE analysis criteria, they were applied in isolation, but their results were compared for the different criteria. They may constitute a potential for the use of TEOAE in schoolchildren screening as well, as is already the case for the neonatal public, or even show changes in the results of diagnostic accuracy from the combination of procedures.

The pass/fail criterion interferes with the results found; other studies involving preschoolchildren and schoolchildren showed a SNR higher than or equal to $3 \mathrm{~dB}$ in all frequency bands and reproducibility higher than $50 \%{ }^{25}$, similar to criterion $A$ and the SNR ratio of the four criteria assessed in this investigation. An 
analysis of different pass/fail criteria in neonatal hearing screening found the presence of response in two or three frequency bands as a valid criterion ${ }^{16}$, different from that found for the sample of this study with schoolchildren. Although they are different populations, the studies with neonates explore more these aspects of the analysis of the criteria to define the presence and absence of the response, as a result of the pass/fail parameter. It is inferred that this difference in results from the pass criterion with presence of response in all bands or two to three frequency bands is probably due to the high presence in neonates of absence or the decreased SNR ratio in the frequency bands of 1000 and $1500 \mathrm{~Hz}$, because of both anatomical issues in the external acoustic meatus of neonates and possible conductive alterations in these structures in the first year of the baby's life. It was also observed a lower mean SNR ratio in the frequency of $1000 \mathrm{~Hz}$, corroborating what is evidenced in neonates and children. Such changes that occur from conductive alterations may also occur among schoolchildren, showing the possibility of adopting this criterion for screening in the school environment.

Quickscreen recording has a short recording window with shorter length, and can be used for fast detection of valid TEOAE in neonates. Compared to other linear and non-linear protocols, this mode had lower reproducibility, especially from 1 to $1.4 \mathrm{kHz}$, with the greatest difference observed in $1 \mathrm{kHz}^{14}$. Another study, also with neonates, used Quickscreen capture as an inclusion criterion for cochlear function screening research ${ }^{21}$. In turn, another study conducted with adults found a significantly higher SNR ratio in the Quickscreen mode in 1,2 and $4 \mathrm{kHz}^{26}$. However, it is important to underline that these studies were conducted with school-aged participants and used different equipment from that used in the current work. Nevertheless, the application of different screening analysis criteria applied and studied in neonates is an important factor to be considered also in schoolchildren, thus discussing the impact of variations in the criteria on the final screening result. Therefore, Quickscreen capture is an important point to be investigated in new studies, mainly for its fast application and due to the fact that this is an aspect to be considered in a school hearing screening program.

Research with Slovakian schoolchildren showed signal-to-noise ratio means lower than $3 \mathrm{~dB}$ in $1000 \mathrm{~Hz}$ and $4000 \mathrm{~Hz}^{27}$, which reiterates the possibility of using SNR ratios lower than $3 \mathrm{~dB}$ in criteria $\mathrm{B}, \mathrm{C}$ and $\mathrm{D}$. It is important to underline that the study by Pavlovcinova and collaborators (2010) ${ }^{27}$ used a diagnostic equipment, while the current study used a portable equipment aimed at performing screening, which may lead to different results. In another study ${ }^{28}$, preschoolchildren and schoolchildren were assessed with TEOAE and pass/fail criterion of SNR ratio higher than $3 \mathrm{~dB}$ in three bands of the five tested frequency bands, similar to criteria $B$ or $C$ in this research, agreeing with the results found here and corroborating the adoption of criterion $\mathrm{C}$.

In this study, the use of $3 \mathrm{~dB}$ revealed a low rate of false positive, high effectiveness and high predictive value, while it is already proposed in the literature that the SNR ratio starting at $1 \mathrm{~dB}$ can improve these results $^{29}$. This partial agreement may be justified due to the wider age group of the current sample. There is greater evidence of conductive hearing loss in children of this age group ${ }^{5}$, besides the possibility of hearing loss in adolescents due to non-occupational noise exposure ${ }^{30}$; therefore, a study that contemplates these two audiences, with probable etiologies of different hearing alterations, points out other results. Among conductive alterations in childhood, otitis media with effusion is frequent and should be considered in hearing health programs. Despite the possible differences between the most frequent hearing impairments in younger children or adolescents, it was not the goal of this study to compare the different criteria between these age groups. However, it is possible that the TEOAE protocols may be adjusted according to age characteristics, a hypothesis that should be investigated in further studies.

The relationships between TEOAE and conductive alterations have been investigated. A Brazilian study that compared TEOAE and distortion product responses in schoolchildren with and without a history of otitis media found that the responses were more likely to be absent and with statistically smaller amplitude in the group with a history of otitis media ${ }^{31}$. A previous study diagnosing otitis media with effusion assessed TEOAE capture with SNR ratio equal to or higher than $6 \mathrm{~dB}$ in four of the five tested frequency bands ${ }^{10}$. Another study $^{32}$ on middle ear alterations used SNR ratio equal to or higher than $3 \mathrm{~dB}$ as a criterion in three of the five tested frequency bands, similar to criteria $B$ and $C$. The absence of TEOAE found was highly related to middle ear alterations ${ }^{32}$. This difference in criteria in specific audiological alterations also points to the importance of new studies with different TEOAE pass/fail criteria, 
since schoolchildren may reveal a diversity of audiological alterations, which should be screened ${ }^{8}$.

The better measurements of diagnostic accuracy found in Criterion $\mathrm{C}$ may be justified not only by the sample characteristics, but also by the TEOAE characteristics. Because they use a broad stimulus such as the click, the response of the Outer Hair Cells' functionality will also be broad, and the absence of response in a frequency band is not a guarantee of hearing loss in that band. Because of these physiological characteristics, the best diagnostic measurements can be justified in the criterion that considers the response in three non-consecutive frequency bands.

It has been recommended that actions be taken to reduce the impact of disabling hearing loss, such as the prevention and identification of hearing loss and ear diseases ${ }^{33}$. In this context, hearing screening plays an important role, as it can be applied on a large scale in asymptomatic populations, thus allowing the identification of these alterations, with a view to carrying out other stages of hearing screening programs.

Hearing screening in schoolchildren is considered cost-effective $^{34}$, but there is no determined protocol for its accomplishment around the world ${ }^{35}$, which gains particular emphasis with TEOAE, already widely used in the country with neonatal hearing screening. They can be used in the initial stage of a school hearing screening program, as an identification stage, which will help not only to determine the prevalence and incidence of hearing loss, but may also contribute to the follow-up of these schoolchildren in the education and health network.

However, despite the relevance of the prevalence and factors associated with hearing loss in schoolchildren ${ }^{4}$, the study of effective and economical methods seems to be a reality in places where hearing health promotion is not a priority and hearing screening is not routine, as in other countries. Seen in these terms, studies and discussions of methods that include technological innovation, such as hearing screening apps $^{36}$ or automated hearing threshold setting ${ }^{37}$, should also be carried out to fit local health realities. In this sense, the inclusion of screening as part of a hearing health program ${ }^{8}$ requires the selection of a procedure that best suits the living and health conditions of the involved population. School children who undergo a hearing screening with TEOAE have a high probability of not having hearing loss ${ }^{12}$, which is further highlighted by the common conditions in this age group of middle ear complications ${ }^{3}$, upper airway infection ${ }^{4}$, cerumen accumulation ${ }^{5,6}$ and non-occupational noise exposure $^{30}$. All these conditions can be prevented to avoid hearing loss, for example, by means of hearing health education actions. However, if these complications are not prevented or identified through hearing screening with a method that is appropriate for this population, the schoolchild will have a reduced chance of intervention at an optimal time that minimizes such negative impacts.

The sample loss in this study may have interfered with the results, as observed by the confidence intervals and predictive values, which impact the external validity of the conclusions. The comparison with results from other studies available in the pertinent literature was limited by the availability of research with equipment similar to that used to capture the TEOAE data in the current study. In addition, the Quickscreen mode used in this investigation is rare in hearing screening studies. Despite the limitations, the results of this study point to the applicability of TEOAE as a screening procedure in schoolchildren. Therefore, it is suggested that further research be conducted comparing not only the different TEOAE criteria, but also the possibility of combining different hearing screening tests, in order to also increase the specificity. Based on the results, it is suggested to adopt criterion $C$ when the TEOAE resources are used alone in the identification of hearing loss in schoolchildren. The application of hearing screening in a hearing health program also contributes to promote hearing health education actions that can contribute positively to the reduction of hearing loss cases.

\section{CONCLUSION}

Among the criteria assessed in this study, criterion $C$ (signal-to-noise ratio equal to or higher than $3 \mathrm{~dB}$ in three tested non-consecutive frequency bands) had sensitivity and specificity higher than $70 \%$ and accuracy of $82.85 \%$, being considered the best option as a criterion for the isolated use of transient emissions as a hearing screening procedure in schoolchildren.

\section{REFERENCES}

1. WHO, World and Health Organization. Deafness and hearing loss [homepage on the internet]. 2018. [accessed 2021 mar 5]. Available at: https:// www.who.int/news-room/fact-sheets/detail/ deafness-and-hearing-loss 
2. Stevens G, Flaxman S, Brunskill E, Mascarenhas $M$, Mathers $C D$, Finucane M. Global and regional hearing impairment prevalence: an analysis of 42 studies in 29 countries. EUR J Public Health. 2011;23(1):1-7. DOI: 10.1093/eurpub/ckr176.

3. Balen SA, Debiasi TF, Pagnossim DF, Broca VS, Roggia SM, Gondim LM. Caracterização da audição de crianças em um Estudo de Base Populacional no Município de Itajaí/SC. Arq. Int. Otorrinolaringol. 2009;13(4):372-80.

4. Nunes ADS, Balen AS, Souza DLB, Barbosa IR. Prevalence of hearing loss and associated factors in school-age individuals in an urban area of Northeast Brazil. Int Arch Otorhinolaryngol. 2020;24(3):330-7. DOI: 10.1055/s-0039-3400507. ISSN 1809-9777.

5. Al-Rowaily MA, AlFayez Al, AlJomiey MS, AlBadr AM, Abolfotouh MA. Hearing impairments among Saudi preschool children. Int J Pediatr Otorhinolaryngol. 2012;76(11):1674-7. doi:10.1016/j.jporl.2012.08.004

6. Tarafder $\mathrm{KH}$, Akhtar $\mathrm{N}$, Zaman $\mathrm{MM}$, Rasel MA, Bhuiyan MR, Datta PG. Disabling hearing impairment in the Bangladeshi population. J Laryngol Otol. 2015;129(2):126-35.

7. American Academy of Audiology. Childhood hearing screening guideline. [homepage on the internet]. September 2011. [accessed $2021 \mathrm{mar}$ 5]. Available at: http://www.cdc.gov/ncbddd/ hearingloss/documents/AAA_Childhood\%20 Hearing\%20Guidelines_2011.pdf

8. Lacerda ABM, Gondim LMA. Hearing health programs for schoolchildren. J Otolaryngol \& Rhinol. 2019;1(4):1-3. DOI: 10.33552/ OJOR.2019.01.000519.

9. Nunes $A D$, Silva $C R$, Balen $S A$, Souza $D L$, Barbosa IR. Prevalence of hearing impairment and associated factors in school-aged children and adolescents: a systematic review. Braz J Otorhinolaryngol. 2019;85(2):244-53. DOI: 10.1016/j.bjorl.2018.10.009

10. Balatsouras DG, Koukoutsis G, Ganelis $P$, Korres GS, Aspris A, Kaberos A. Transiently evoked otoacoustic emissions in children with otitis media with effusion. Int. J. Otorhinolaryng. 2012;2012:269203. DOI: 10.1155/2012/269203

11. Prieve BA, Schooling T, Venediktov R, Franceschini $\mathrm{N}$. An evidence-based systematic review on the diagnostic accuracy of hearing screening instruments for preschool- and school-age children. Am J Audiol. 2015;24(2):250-67. DOI:10.1044/2015_AJA-14-0065

12. Yin L, Bottrell C, Clarke N, Shacks J, Poulsen MK. Otoacoustic emissions: a valid, eficiente first-line hearing screen for preschool children. J Sch Health. 2009;79(4):147-52.

13. Cedars E, Kriss H, Lazar AA, Chan C, Chan DK. Use of otoacoustic emissions to improve outcomes and reduce disparities in a community preschool hearing screening program. Plos One. 2018;13(12):e0208050.

14. Jedrzejczak WW, Kochanek K, Sliwa L, Pilka E, Piotrowska A, Sharynski H. Chirp-evoked otoacoustic emissions in children. Int $\mathrm{J}$ Pediatr Otorhinolaryngol. 2013;77(1):101-6. DOI: 10.1016/j. ijporl.2012.10.005.

15. Liu J, Wang N. Effect of age on click-evoked otoacoustic emission: a systematic review. Neural Regen Res. 2012;7(11):853-61.

16. Côrtes-Andrade IF, Bento DV, Lewis DR. Transient evoked otoacoustic emissions (TEOE): newborn hearing screening program protocols. Rev. CEFAC. 2013;15(3):521-7. DOI: 10.1590/ S1516-18462012005000062

17. WHO, World Health Organization. Basic ear and hearing care resource [homepage on the internet] 2020 [accessed $2021 \mathrm{feb} 20$ ]. Available at: https:// apps.who.int/iris/rest/bitstreams/1270187/retrieve

18. Menegotto $\mathrm{IH}$. Logoaudiometria básica. In: Bevilacqua MC, Martinez MAN, Balen SA, Pupo AC, Reis ACMB, Frota S, editors. Tratado de Audiologia, 1a ed. São Paulo: GEN, 2011. p.81-99.

19. Jerger J. Clinical experience with impedance audiometry. Arch Otolaryngol. 1970;92:311.

20. Otoport manual. Otoport DP+TE/DP User Manual Issue 5. Otodynamics Ltd. November; 2009.

21. Carvallo RMM, Sanches SGG, Ibidi SM, Soares JC, Durante AS. Efferent inhibition of otoacoustic emissions in preterm neonates. Braz J Otorhinolaryngol. 2015;81(5):491-7.

22. Medeiros MMC, Abreu MM. Epidemiologia clínica. In: Rouquayrol MZ, Silva MGC, editors. Epidemiologia e Saúde, 13a ed. Rio de Janeiro: MedBook, 2013. p.149-75.

23. Feder KP, Michaud D, McNamee J, Fitzpatrick E, Ramage-Morin $\mathrm{P}$, Beauregard $\mathrm{Y}$. Prevalence of hearing loss among a representative sample of Canadian children and adolescents, 3 to 19 years of age. Ear and hearing. 2017;38(1):7-20. DOI: 10.1097/AUD. 0000000000000345 
24. Ramkumar V, John KR, Selvakumar K, Vanaja CS, Nagarajan R, Hall JW. Cost and outcome of a community-based paediatric hearing screening programme in rural India with application of tele-audiology for follow-up diagnostic hearing assessment. Int J Audiol. 2018;57(6):407-14. DOI:1 0.1080/14992027.2018.1442592

25. Norton SJ, Widen JE. Evoked otoacoustic emissions in normal-hearing infants and children: emerging data and issues. Ear Hear. 1990;11(2):121-7.

26. Kei J, Sockalingam R, Holloway C, Agyik A, Brinin C, Baine D. Transient evoked otoacoustic emissions in adults: a comparison between two test protocols. J Am Acad Audiol. 2003;14(10):563-73. DOI:10.3766/jaaa.14.10.5

27. Pavlovcinová G, Jakubíková J, Trnovec $\mathrm{T}$, Lancz K, Wimmerová S, Sovcíková E et al. A normative study of otoacoustic emissions, ear asymmetry, and gender effect in healthy schoolchildren in Slovakia. Int $\mathrm{J}$ Pediatr Otorhinolaryngol. 2010;74(2):173-7. DOI:10.1016/j.ijporl.2009.11.002

28. Jedrzejczak WW, Hatzopoulos S, Sliwa E, Pilka E. Otoacoustic emissions in neonates measured with different acquisition protocols. Int. J. Pediat. Otorhinolaryng. 2013;76(3):382-7. DOI: 10.1016/j. ijporl.2011.12.016.

29. Driscoll C, Kei J, McPherson B. Outcomes of transient evoked otoacoustic emission testing in 6-year-old school children: a comparison with pure tone screening and tympanometry. Int $\mathrm{J}$ Pediatr Otorhinolaryngol. 2001;57(1):67-76.

30. Marques APC, Miranda Filho AL, Monteiro GTR. Prevalence of hearing loss in adolescents and young adults as a result of social noise exposure: meta-analysis. Rev. CEFAC. 2015;17(6):2056-64. DOI: 10.1590/1982-021620151761115

31. Sanfins MD, Bertazolli LF, Skarzynski PH, Skarzynska MB, Donadon C, Colella-Santos MF. Otoacoustic emissions in children with long-term middle ear disease. Life (Basel). 2020;10(11):287. DOI:10.3390/life10110287

32. Georgalas C, Xenellis J, Davilis D, Tzangaraoulakis A, Ferekidis E. Screening for hearing loss and middle-ear effusion in school-age children, using transient evoked otoacoustic emissions: a feasibility study. J Laryngol Otol. 2008;122(12):1299-304.

33. Graydon K, Waterworth C, Miller H, Gunasekera $\mathrm{H}$. Global burden of hearing impairment and ear disease. J Laryngol Otol. 2019;133(1):18-25. DOI: $10.1017 /$ S0022215118001275
34. Yong M, Liang J, Ballreich J, Lea J, Westerberg BD, Emmett SD. Cost-effectiveness of school hearing screening programs: a scoping review. Otolaryngol Head Neck Surg. 2020;162(6):826-38. DOI: 10.1177/0194599820913507.

35. Yong M, Panth N, McMahon CM, Thorne PR, Emmett SD. How the world's children hear: a narrative review of school hearing screening programs globally. OTO Open. 2020;4(2):2473974X20923580. DOI: 10.1177/2473974X20923580.

36. Mahomed-Asmail F, Swanepoel de W, Eikelboom $\mathrm{RH}$. Diagnostic hearing assessment in schools: validity and time efficiency of automated audiometry. J Am Acad Audiol. 2016;27(1):42-8. doi:10.3766/jaaa.15041

37. Hong SM, Park I-S, Kim YB, Hong SJ, Lee B. Analysis of the prevalence of and factors associated with hearing loss in Korean adolescents. PLoS ONE. 2016;11(8):e0159981. DOI:10.1371/journal. pone.0159981 